\title{
Morphological Transformation In Poor Lighting Images For Image Contrast Enhancement
}

\section{Gutta Srinivas Rao ${ }^{a^{*}}$, Atluri Srikrishna ${ }^{b}$}

\author{
${ }^{a *}$ Department of Information Technology, RVR \& JC College of Engineering, Guntur, Andhra Pradesh, India. \\ E-mail: gsr.rvr@gmail.com \\ ${ }^{\mathrm{b}}$ Department of Information Technology, RVR \& JC College of Engineering, Guntur, Andhra Pradesh, India. \\ E-mail: atlurisrikrishna@yahoo.com
}

Article History: Received: 11 January 2021; Accepted: 27 February 2021; Published online: 5 April 2021

\begin{abstract}
The paper presents a novel algorithm for the computation of the image decomposition using a morphological filter with reconstruction. The target applications are image contrast enhancement especially those with high dynamic content. Both bright and dark regions contrast enhancement were considered. A new hardware efficient implementation of decomposition is presented. Following decomposition in 5 levels of detail a local contrast enhancement is performed. The new reconstruction algorithm and its hardware implementation as proposed is shown to be independent on structural element size and that it results in a predictable time frame operation. A mixed schematic and VHDL/Verilog description of the decomposition filters was synthesized and results show far higher speed performance compared with solutions found in recent literature.

Image organizing strategies are essentially required to the pictures captured by satellites. The grey levels in the image captured by satellites need to be normalized for improving picture contrast. The pictures captured by satellites are generally poor in quality and the contrast levels are also very less. In this manuscript a differentiation approach dependent on scientific morphology called rotational morphological transformations are proposed using Modified Opening by Reconstruction Method (MORM). This methodology upgrade pictures with poor contrast and unable to recognize the objects in the image. In the images with poor lightening, morphological operations are performed to improve its clarity and contrast. Picture enhancement has been done by applying morphological operations on the satellite images considered. The technique utilizes data from picture by squares, while the morphological methods change the strategy using the contrast enhancement activity, which is utilized to characterize the multi-foundation poor lightening pictures. The total picture handling procedure is implemented utilizing MATLAB reproduction model. The MORM method is compared with the traditional methods and the results show that the proposed method is better in improving the accuracy rate.
\end{abstract}

Keywords: Image Contrast Enhancement, Satellite Images, Blocks, Pixel Intensity Ratio, Gray Level Balancing, Morphological Operations.

\section{Introduction}

Morphological channels comprise an advantageous and effective strategy for differentiate improvement in the quality of images. The different computational choices are analyzed in various levels. It is indicated that the iterative mono dimensional auxiliary component approach conveys lower execution giving ongoing limitations when contrasted with various size secondary components[1]. A single scale various measurement organizing component variation was picked as suitable for contrast enhancement[2].

It is realized that the significant trouble in utilizing morphological channels continuously in image contrast enhancement applications is their natural unpredictability and high calculation cost. Progressively in image quality enhancement applications, the computational multifaceted nature issue is significantly all the more a limitation[3]. High unique pictures captured from long range have poor contrast with less quality. Both brighter pixels and dull pixels are identified and the poor contrast pixel quality enhancement is the key task in the proposed method. Ordinarily the complexity is improved by nearby expansion of the force scale in the area of scheme. The coefficients for scale expansion yield best outcomes[4]. Morphological channels comprise an advantageous and productive strategy for differentiate upgrade.

The main goal of picture enhancement is to acquire important data from the discernment/scene[5]. The principle point of upgrade of pictures is differentiate control, decrease of noise levels from the picture, edges honing, sifting, base exclusion, and so forth. The strategy is very useful to extricate noise from the pictures[6][7]. Upgrade should be possible by utilizing morphological methods to get the more improved results. In the current examination, the differentiation improvement of picture is finished by applying morphological tasks like with the assistance of various organizing elements[8]. The proposed method is used for enhancing the contrast levels of 
the pixels by dividing the images into blocks and applying the method into specific blocks. The Figure 1 represents the process.
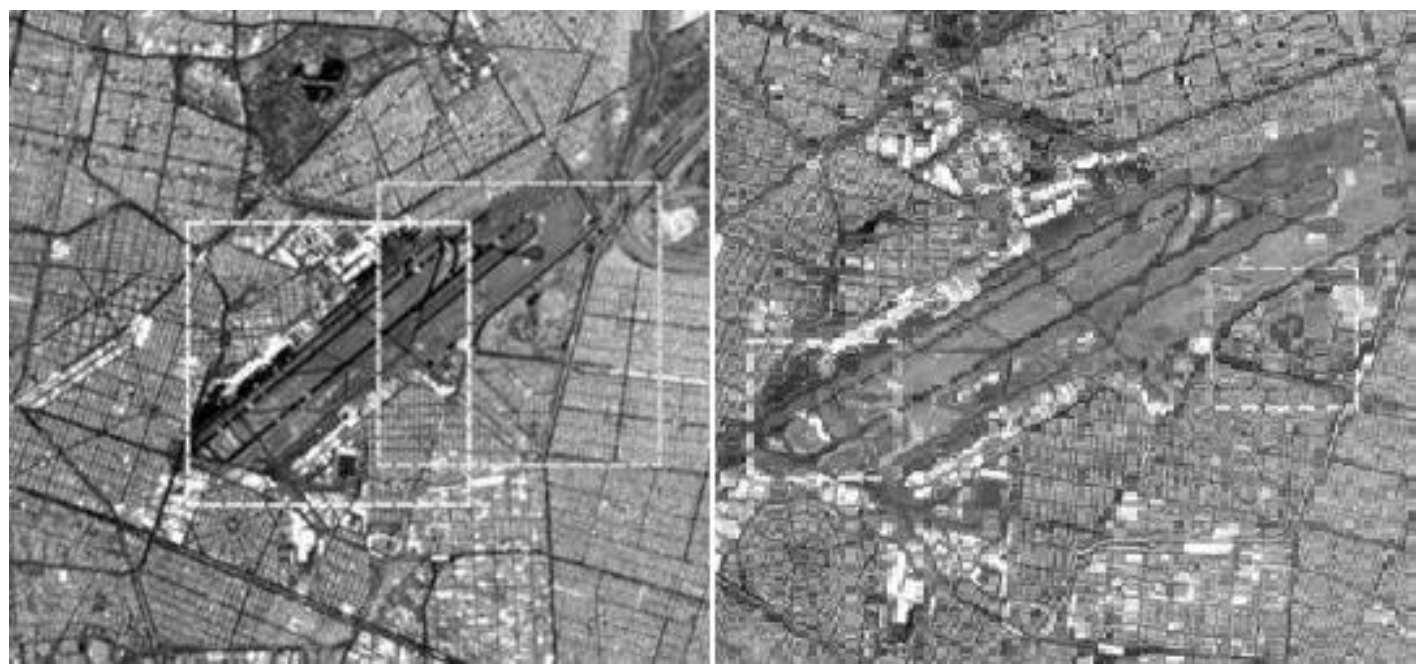

Fig. 1. Image to Blocks Division

Picture quality improvement is a challenging task in satellite image handling strategies. Picture arrangement infers auxiliary features, which are then numerically evaluated by picture examination. Complexity raise assumes a significant job in picture handling; it improves auxiliary features that are not really perceivable to the human eye and permits programmed extraction of those features[9]. Various differentiation upgrade techniques have been accounted for including histogram strategies, and spatial space and recurrence area sifting. In any case, these strategies will in general improve whole structures in a satellite picture without segregation[10]. To viably perceive an area of image, explicit objective structures must be improved while encompassing pixels that stay unmodified.

Morphology works by a progression of morphological tasks, which utilize little pictures called organizing components. The organizing component goes about as a moving test that examples every pixel of the picture[11]. Since the organizing component moves a fixed way over the picture, some perplexing pictures specifically, those whose auxiliary pixels contain an assortment of directional characters that may not be appropriate.

The image contrast enhancement issue in computerized pictures can be drawn nearer from different philosophies, among which is numerical morphology[12]. Such administrators comprise in agreement to some closeness basis, in choosing for each purpose of the investigated picture, another dark level between two examples[13]. Despite the fact that morphological difference has been generally considered, there are no approaches, from the perspective prepared to do all the while normalizing and improving the balance in pictures with poor lighting[14][15]. On the opposite side, one of the most widely recognized strategies in picture contrast enhancement is to improve dull pixels with utilization of nonlinear capacities, for example, logarithm or power capacities ; something else, a strategy that works in the recurrence space is the homomorphism channel[16]. Notwithstanding, the principle detriment of histogram balance is that the worldwide properties of the picture can't be appropriately applied in a nearby setting, as often as possible delivering a method for quality enhancement[17].

\section{Literature Survey}

A. Agrawal et al [1] proposed a methodology called for image contrast enhancement. Pictures disturbed by disorder have two principle attributes: high brightness and low concentration. In this way, a weighted entirety of a picture brightness and immersion is utilized to depict the darkness level. Barometrical light can be evaluated by a similar standard, while a little rectification is required when pictures contain over- bright pixels. After the two weighted maps are developed, nearby insights of the seriousness map are applied in picture dull pixels. Results are investigated and contrasted subjectively and quantitatively with four best in class techniques.

A.V. Oppenheim et al [2] proposed to manage outstanding load varieties that are brought about by picture handling methods utilizing a intellectual methodology. Rather than planning the controller for the most pessimistic scenario, interminably adjust the inspecting time of the controller on the real case at run-time. In that manner, the normal testing time frame is improved concerning a image structure. 
B.I. Justusson et al [5] discussed the use of estimated registering in equipment based PC vision. Receiving the ideas of versatile pressure and inexact quantization, C. Chen et al [6] proposed a picture sensor structure for PC vision undertakings that altogether moves the pixel organize scheme, sub sampled picture information that accomplish a significant decrease in vitality while keeping the classification exactness of regular DNN datasets, for example, the CIFAR-10, inside satisfactory levels.

J. Baili et al [8] identified that assumed figuring can be used in spaces other than AI or picture preparing and showed a start to finish contextual investigation on the space of biometric security frameworks focusing on an iris checking application. The test in an iris filter application is that it is a multidimensional issue to inexact, as more than one calculations should be associated with a pipelined design so as to deliver results and have the option to measure the effect of estimation. The proposed methodology utilizes a four-arrange pipeline with a camera sensor stage to acquire the picture, a center arrangement so as to pick the casing with the best core interest among resulting outlines, an iris division arrangement that figures the middle purposes of the iris also, and finally a standardization organized method that delivers the iris signature.

J. Kosecka et al [9] exhibited a calculation in which dull zones have been considered by applying organizing segments, accordingly two unique features having light and dark zones at each scale which are used to acquire the last light and dull regions of a picture. At that point enhancement is finished by extending the complexity between various pixels of the picture.

J. Serra et.al. [11] proposed a calculation dependent on feature extraction of the pictures, a novel morphological based homomorphic partition which has been recommended for improvement. This strategy depends on the deterioration of a picture into various sub-groups of morphology followed by homomorphic sifting and afterward calculation dependent on differential development is utilized to decide best increase and organizing segments for each sub-band.

K. Grabowski et al [13] build up a method utilizing edge-discovery. The places of the edges are recognized first utilizing inclination based administrators, at that point morphological sifting is applied. N. C. Gallagher et al [15] proposed a strategy for improving the form of pictures as improvement of coronary route based multiscale analyzations. Gabor wavelet is utilized for the recognition of the width of the vessel on every single pixel in a picture. At that point, a gathering of morphological opening tasks are applied on the picture dependent on the evaluated scales. At last, this assessed foundation is subtracted from the first angiogram.

P. Maragos et al [16] proposed techniques comprising of two stages: procedure called un-sharp covering is utilized to improve the limits and edges of the pictures. Interestingly improvement step, a $3 \times 3$ picture is utilized with qualities that go over the limit and stay unaltered though different pixels are remapped. The outcomes from applying this methodology on dim scale; shading and restorative pictures show that the procedure is vigorous and ready to recuperate even too dull pictures from obscuring and darkness.

P. Maragos et al. [17] proposed a calculation to improve the edge structure for certifiable element extraction. Morphological expansion and disintegration tasks are applied on dim scale pictures and morphological top-cap change is utilized for improving contrast levels. The test results appeared that the calculation can successfully lessen the complexity, lessen the commotion and fills the holes between the messed up spans.

\section{Proposed Method}

The satellite images are considered for contrast enhancement and the process of enhancing the contrast of the images are done in several steps. Initially when a image is considered, the median filtering is applied for removal of noise values in the image. The median filter $\beta$ is calculated as

$$
\beta_{\text {med }}^{2}=\frac{1}{4 p t^{2}(n)} \approx \frac{\pi_{i}^{2}}{n+\frac{\pi}{2}-1} \cdot \frac{\pi}{2}
$$

Here $\mathrm{p}$ is the pixels in the image and $\mathrm{t}$ is the intensity level, $\mathrm{n}$ is the total pixels in the image. The pixel intensity levels 'Im' are calculated using the equation below.

$$
\left(\operatorname{Im}_{\text {out }}(i)-\operatorname{Im}_{\text {out }}(i-1)\right)+c(i+1)=\left(\operatorname{Im}_{\text {out }}(i+1)-\operatorname{Im}_{\text {out }}(i)\right) c(i)
$$

A weighting capacity is utilized to deal with situations when the brighter pixels are zero, it is resolved as 


$$
R_{i, j}=\exp \left(\frac{-\left(\operatorname{Im}_{i n}(i)-\operatorname{Im}_{i n}(j)\right)^{2}}{\left(\frac{2 \beta^{2}}{2}\right) * \mathrm{i}}\right) .
$$

The equality condition used for balancing the pixels ratio is calculated as

$$
x_{i, i-1}\left(\operatorname{Im}_{\text {out }}(i)-\operatorname{Im}_{\text {out }}(i-1)\right)=x_{i+1, i}\left(\operatorname{Im}_{\text {out }}(i+1)-\operatorname{Im}_{\text {out }, i}\right) c(i) /\left(\frac{2 \beta^{2}}{2}\right) * \mathrm{i}+\mathrm{R}
$$

The morphological operations are applied on the images for enhancing the contrast on the poor quality images. The morphological operations applying structuring of image with same considered size as that of input image.

Morphological opening is $\gamma_{\mu B}(f)(x)$ and Morphological closing is $\varphi_{\mu B}(f)(x)$

$$
\begin{gathered}
\gamma_{\mu \bar{B}}(f)(x)=\delta_{\mu \bar{B}}\left(\varepsilon_{\mu \bar{B}}(f)\right)(x) / \operatorname{Im}_{\text {out }} \\
\varphi_{\mu \bar{B}}(f)(x)=\varepsilon_{\mu \bar{B}}\left(\delta_{\mu \bar{B}}(f)\right)(x)+R / c(i+1) *\left(\frac{2 \beta^{2}}{2}\right)
\end{gathered}
$$

Where $\mu$ a homothetic parameter, size is $\mu$ means a square of $(2 \mu+1) \times(2 \mu+1)$ pixels. B is the structuring element of size $3 \times 3$ (here $\mu=1$ ).

For every single pixel $\mathrm{n}$, the base power mi and greatest strength mi esteems are determined mi esteems are utilized to discover the background criteria $\mathrm{i}, \lambda$ in the following way:

$$
\lambda_{i}=\frac{m_{i}+M_{i}}{2} \forall_{i}=1,2, \ldots, n
$$

$\lambda$ is utilized as an edge between clear $(\mathrm{mi}>\lambda)$ and dull $(\mathrm{mi}=\lambda)$ force levels. In view of the estimation of $\mathrm{I}$, the base parameter is chosen. Correspondingly the differentiation upgrade is communicated as pursues:

$$
\Gamma_{\tau_{i}}(f)=\left\{\begin{array}{c}
L_{i} \log (f+1)+M_{i,} f \leq \tau_{i} \\
L_{i} \log (f+1)+m_{i,} \text { Otherwise }
\end{array}\right.
$$

Plainly the base parameter totally is need on the base criteria $\mathrm{I} \lambda$ value. For $\mathrm{I} f=\lambda$, the foundation parameter takes the greatest power value $\mathrm{Mi}$ inside the dissected square, and the base force esteem mi generally. So as to maintain a strategic distance in assurance condition, unit was added to the logarithmic capacity.

$$
\begin{gathered}
\text { Where } L_{i}=\frac{255-m_{i}^{\bar{*}}}{\log (256)} \forall_{i}=1,2, \ldots, n \\
\text { With } m_{i}^{*}=\left\{\begin{array}{l}
m_{i,} f \leq \tau_{i} \\
M_{i,}, f \geq \tau_{i}
\end{array}\right.
\end{gathered}
$$

The proposed Modified Opening by Reconstruction Method enhances the pictures with less contrast and to improve the quality of such kind of images.

\section{Algorithm: Modified Opening by Reconstruction Method (MORM)}

Step 1: Input a Satellite Colored Image

Step 2: The background criteria for total image $\lambda(\mathrm{x})$ is calculated as:

Background criteria $\lambda(\mathrm{x})=\gamma \mu$ (f) (x).

Step 3: The background of the image that gives the local information is calculated as:

$\mathrm{b}(\mathrm{x})=\in 1 \gamma \mu(\mathrm{f})(\mathrm{x})$.

where the structuring element size $\mu=1$

Step 4: Contrast enhancement of the pixels are performed as:

$\varepsilon \gamma \mu$ (f) $=\alpha * k(x) * \log (f+1)+\varepsilon 1 \gamma \mu$ (f) 
- $\quad$ And $\alpha=(1-(\operatorname{avg} / \mathrm{L}))$

avg is the average of the image pixel intensities.

$\mathrm{k}(\mathrm{x})=\mathrm{L}-\varepsilon 1 \gamma \mu(\mathrm{f}) / \log (\mathrm{L}+1)$

where $L=2^{B}-1, B$ is the number of bits used to represent the pixel values.

Step 5: After enhancing the contrast level of the image, the contrast levels are calculated as:

Local contrast value $C(x, y)$ can be computed as

$$
C(x, y)=\frac{|p a|}{|p+a|}+\left(\operatorname{Im}_{i n}(i)-\operatorname{Im}_{i n}(j)\right)^{2}
$$

If the contrast level $\mathrm{C}$ is less than 30\%, then the Contrast Improvement ratio is increased using Step 6.

Step 6: Contrast Improvement Ratio (CIR) is characterized as the proportion of the differentiation of the upgraded and unenhanced pictures inside set of pixels $\mathrm{R}$, as pursues,

$$
C I R=\sum_{(p, q \in R)}|C(p, q)-\bar{C}(p, q)|^{2} / \sum_{(p, q \in R)} C(p, q)^{2} * \mathrm{R}+\left(\frac{2 \beta^{2}}{2}\right)
$$

Where $C(p, q)$ are the nearby differentiation values at $(p, q)$ of the unenhanced and improved pictures, separately. The neighborhood differentiate value $\mathrm{C}(\mathrm{p}, \mathrm{q})$ is figured as

Step 7: Stop.

$$
C(p, q)=|x-a| /|x+a| *\left(\frac{2 \beta^{2}}{2}\right)-\mathrm{R}
$$

The parameters considered to identify the quality of the image are Peak Signal to Noise Ratio (PSNR), Absolute Mean Square Error (AMSE), Entropy, Structural Similarity Index (SSI), Tenengrad. The proposed method when applied on the satellite image dataset, the considered parameters are calculated for recognition of the improvement of the quality of the images.

By considering the luminance signal, the errors are identified. The Peak Signal to Noise Ratio is calculated using equation

$$
M S E=\frac{\sum_{p=1}^{x} \mathrm{p} \sum_{q=1}^{y} \mathrm{q}(\mathrm{T}(p, q)-G(p, q))^{2}}{x * y}
$$

The absolute mean square error is the difference between input and output mean and is calculated as

$$
\text { AMSE }=\frac{1}{G} \sum_{i=1}^{T} \mathrm{G}|p-q|
$$

Entropy of image is the information of the image measurement that a human can identify. It can depict the quality of the image and calculated as

$$
E(p)=-\sum_{x=0}^{255} \mathrm{p}+\mathrm{q} p(x) \log _{2} q(i)
$$

Natural image signals are highly structured. Their pixels exhibit strong dependencies, especially when they are spatially proximate and these dependencies carry important information. The Structural Similarity Index SSI is calculated as

$$
\operatorname{SSI}(p, q)=\frac{(20 p a q+c 1)(2 \pi p q+c 2)}{\left(\sigma_{p}^{2}+\sigma_{q}^{2}+c 1\right)\left(\sigma_{p}^{2}+\sigma_{q}^{2}+c 2\right)}
$$

Tenengrad is a powerful image quality assessment measure in terms of sharpness. Sharpness is a very helpful parameter in photographic image quality factor. It is calculated as 


$$
T g=\sum_{i=1}^{p} g(p, q) \sum_{j=1}^{q} g(p, q)
$$

\section{Results}

The proposed method uses satellite dataset for considering the images and improving the contrast of the poor quality images to enhance the contrast in those images. The Modified Opening by Reconstruction Method considers satellite data from the https://earthexplorer.usgs.gov/ link. The proposed method is implemented in MATLAB 9.4. The proposed method is compared with the traditional methods and the results show that that the proposed method is better than the existing methods.

The proposed method initially applies pre processing for removal of noise values and then the image pixel intensity values are calculated. The image intensity values identified are depicted in Figure 2.

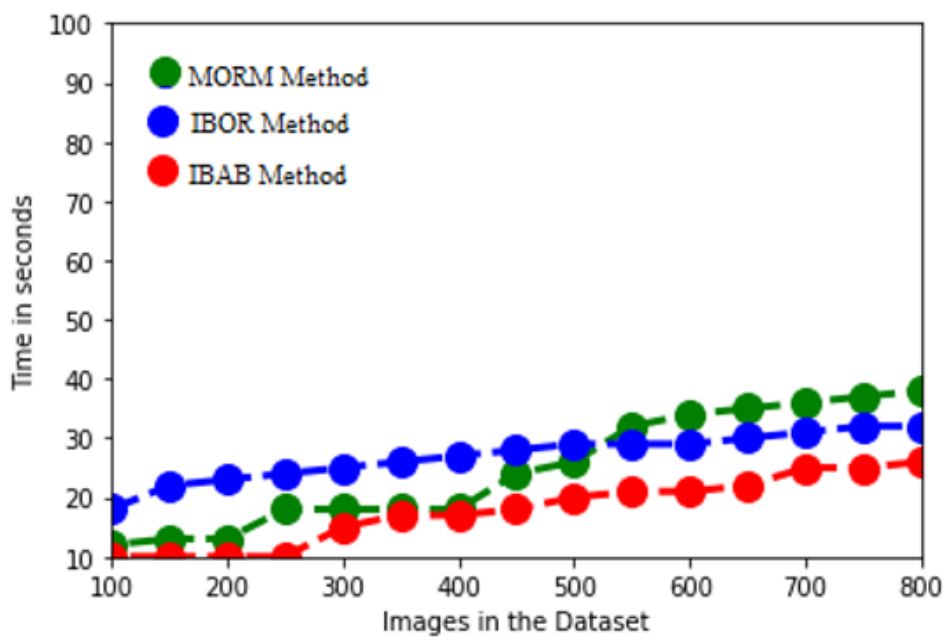

Fig. 2. Image pixel Intensity Values Levels

The proposed MORM method efficiently enhances the dull pixels contrast and improves the quality of the images. The image is divided into blocks and then the block which has dull pixels are applied with the MORM method. The process is depicted in Figure 3.

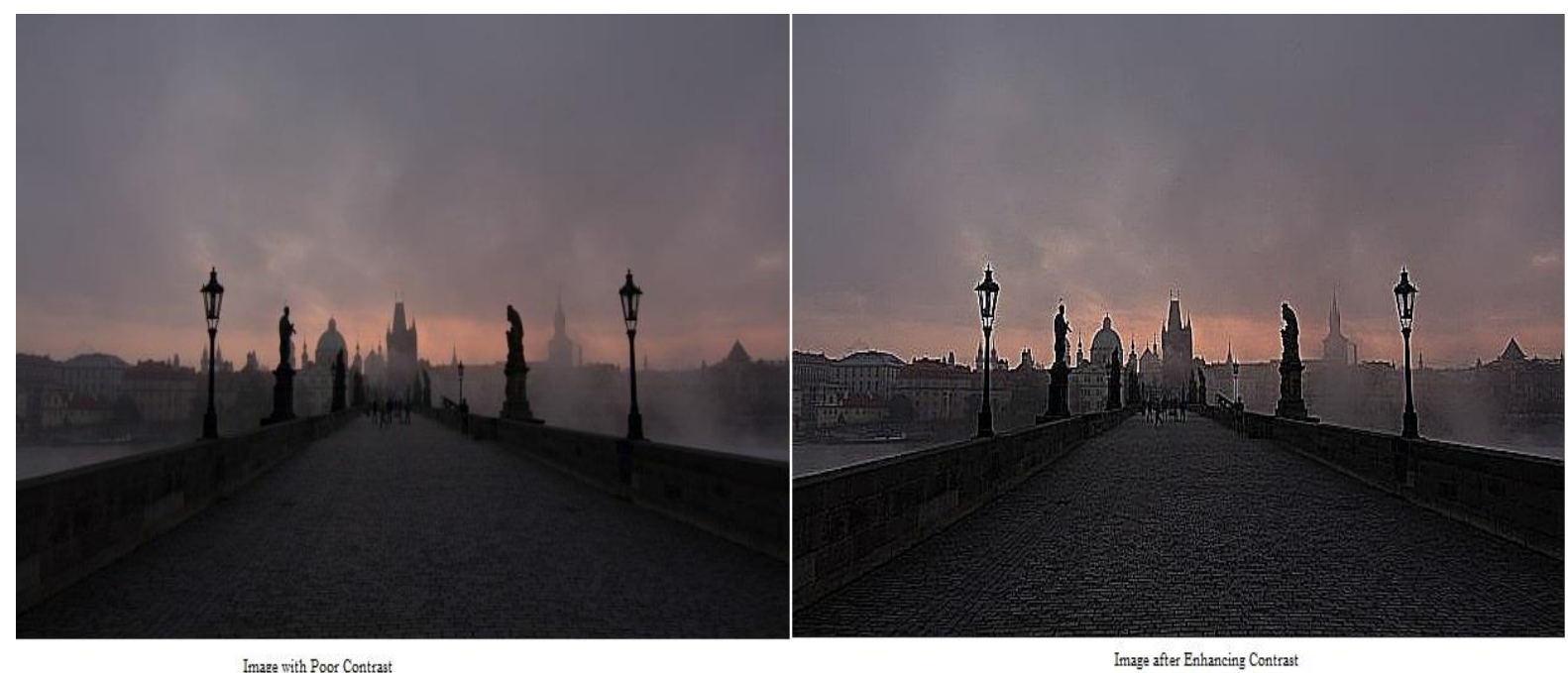

Fig. 3. Image Contrast Enhancement

The proposed method considers the satellite images and then initially the contrast levels are calculated. After calculating the contrast levels, image is divided into block and the pixel intensity values are calculated. The Image pixel enhancement process is done more quickly in the proposed method. The process is contrasted with the traditional methods and depicted in Figure 4. 


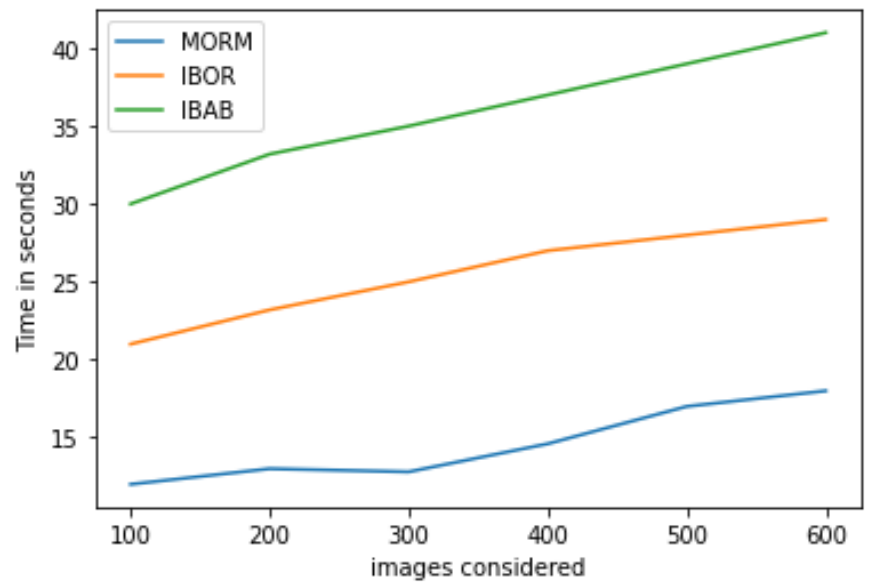

Fig. 4. Image pixel Enhancement Time Levels

The Images after considered from the satellite dataset are applied with the proposed method and then the dull pixels and contrast maintained pixels are separated as blocks and then the MORM method is applied on the dull pixels and the images are represented in Figure 5.

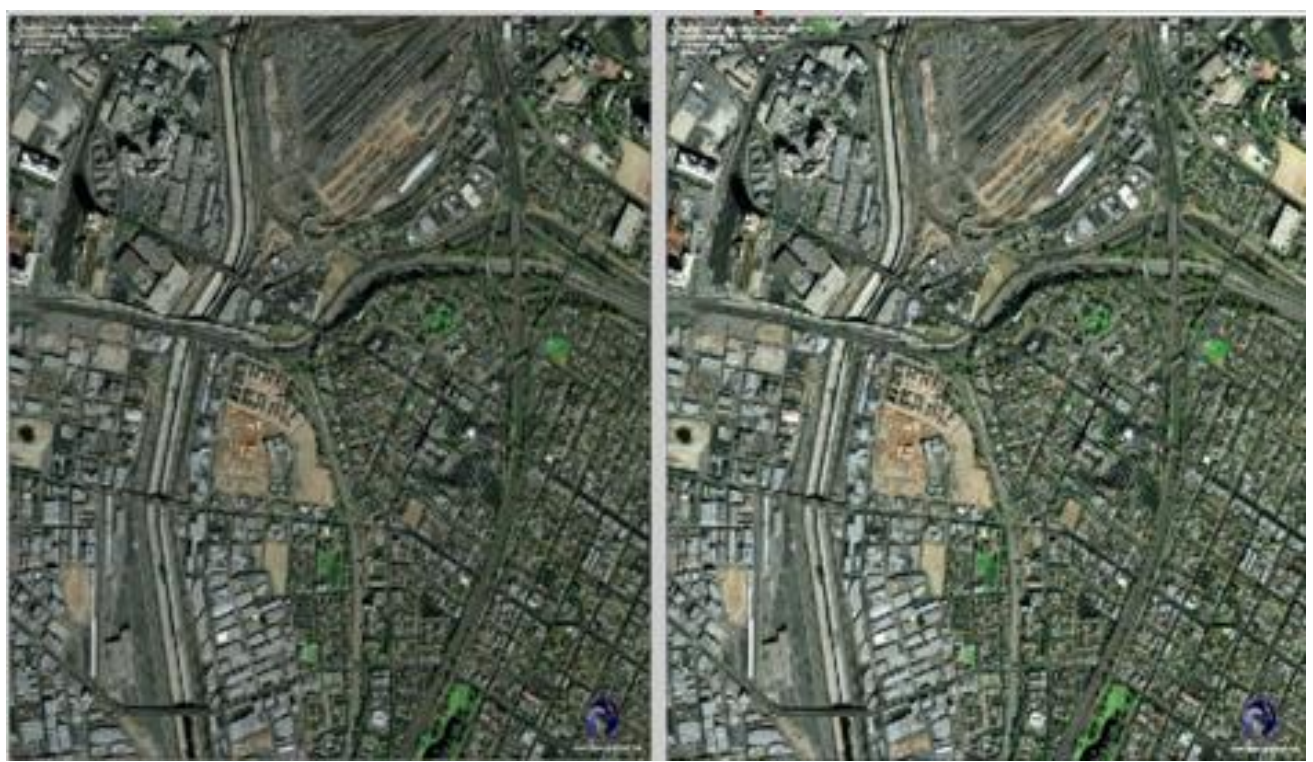

Fig. 5. Enhancing the Dull Pixels

The objects in the image can be clearly detected after the enhancement of contrast levels and then the images are represented in Figure 6.
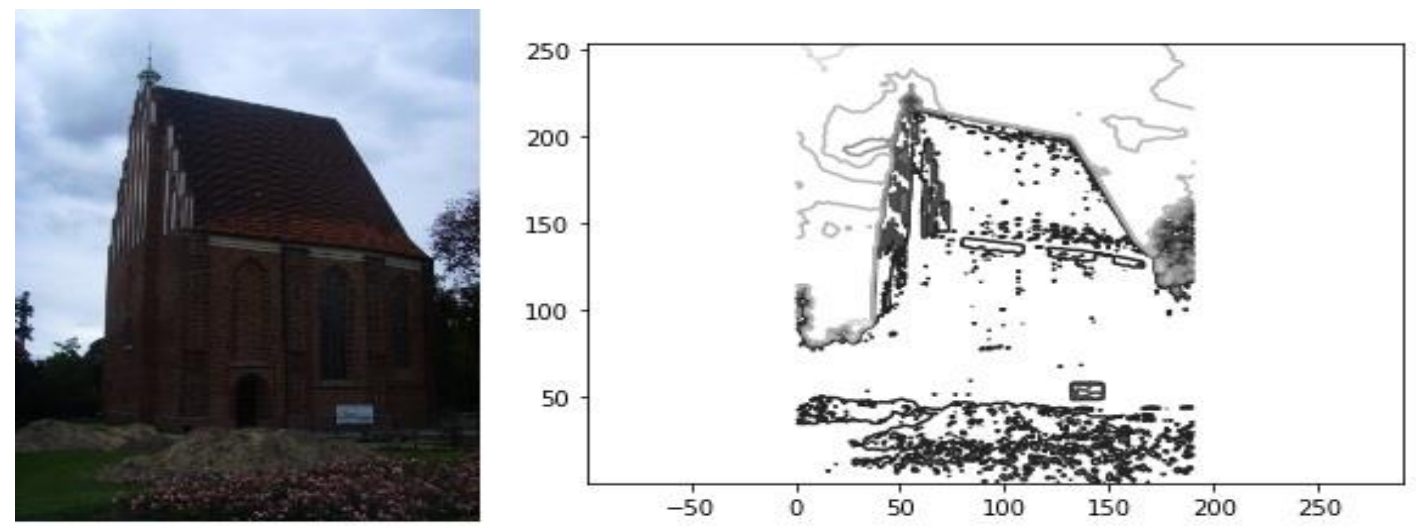

Fig. 6. Object Detection in the Images 
The images having less quality or images that are having full dull pixels are applied with the morphological operations and the objects are identified accurately. The process is depicted in Figure 7.

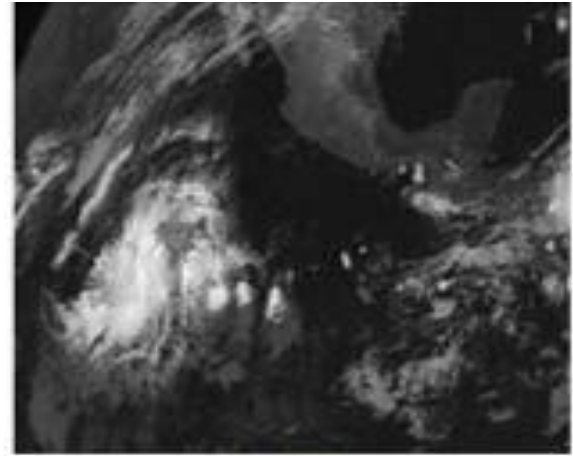

(a)

(c)

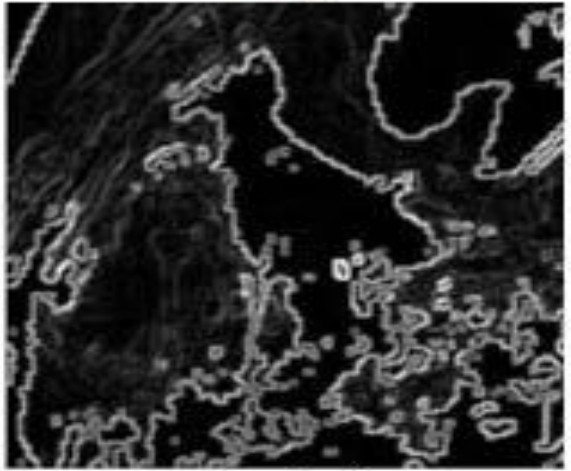

(e)

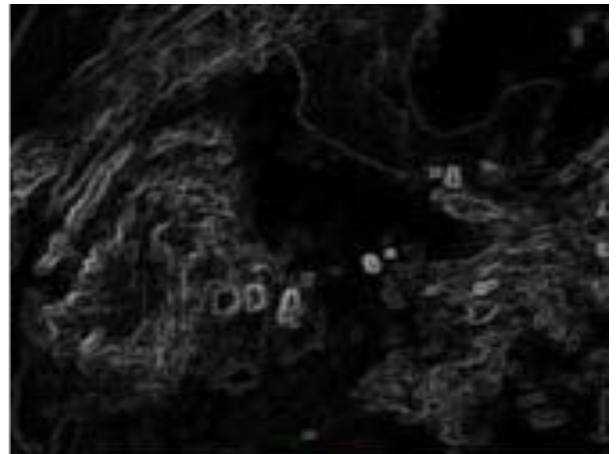

(b)

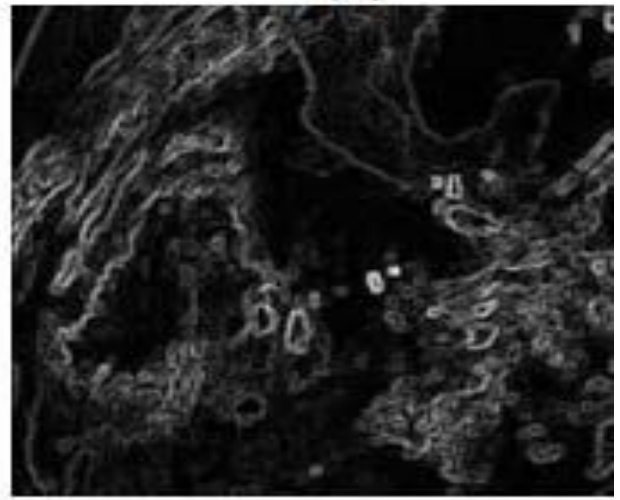

(d)

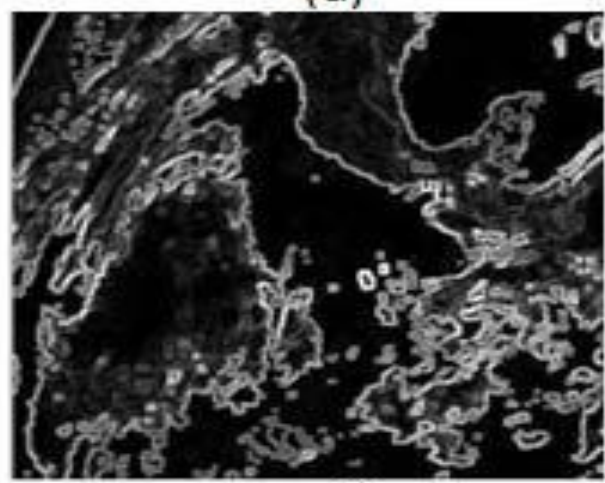

(f)

Fig. 7. Object Identification in Dull Images

The blocks which have dull images are applied with the MORM method for enhancing the contrast of the dull pixels. The table-1 illustrates the method and the contrast level identified.

Table 1. Contrast levels Identified in Images

\begin{tabular}{lll}
\hline \multicolumn{1}{c}{ Method Name } & $\begin{array}{c}\text { Dull Pixels Level } \\
\text { Identified }\end{array}$ & $\begin{array}{c}\text { Enhanced Pixels } \\
\text { Contrast Level }\end{array}$ \\
\hline Image Background Approximation by Blocks (IBAB) & $13 \%$ & $29 \%$ \\
Image Background Determination Using the Opening by & $15 \%$ & $26 \%$ \\
Reconstruction (IBOR) & & $38 \%$ \\
Modified Opening by Reconstruction Method (MORM) & $9 \%$ & \\
\hline
\end{tabular}

The different parameters are calculated and the proposed morphological method when applied on the satellite images, calculated values are depicted in Table 2. 
Table 2. Multiple Parameter Measures

\begin{tabular}{|c|c|c|c|c|c|c|}
\hline \multirow{2}{*}{ Method Name } & \multirow{2}{*}{ PSNR } & \multirow{2}{*}{ AMSE } & \multicolumn{2}{|c|}{ Entropy } & \multirow{2}{*}{ SSI } & \multirow{2}{*}{ Tenengrad } \\
\hline & & & Input & Output & & \\
\hline $\begin{array}{l}\text { Image Background Approximation by Blocks } \\
\text { (IBAB) }\end{array}$ & 22.35 & 14.95 & 5.95 & 6.84 & 0.84 & 846611 \\
\hline $\begin{array}{l}\text { Image Background Determination Using the Opening } \\
\text { by Reconstruction (IBOR) }\end{array}$ & 15.11 & 13.64 & 4.36 & 5.21 & 0.68 & 724156 \\
\hline $\begin{array}{l}\text { Modified Opening by Reconstruction Method } \\
\text { (MORM) }\end{array}$ & 6.12 & 5.05 & 1.56 & 4.65 & 0.39 & 467121 \\
\hline
\end{tabular}

\section{Conclusion}

Image contrast enhancement is a technique applied on satellite images. The images considered from the satellite database are initially calculated with the dull contrast pixels and the images are arranged in blocks. The contrast improvement on the pixels is performed by balancing the pixels of normal and dull regions and then morphological operations are applied on the images and that on the pixels of less contrast and then contrast improvement ratio process is done by improving the contrast of the pixels whose contrast is very less. The MORM method is applied on the dull pixels for improving the contrast ratio to make the image clearer and to improve the quality of the images. The proposed method is contrasted with the traditional methods and the results show that the proposed method is better in improving the image quality and contrast.

\section{References}

6. Mercat, A., Bonnot, J., Pelcat, M., Hamidouche, W., \& Menard, D. (2017). Exploiting computation skip to reduce energy consumption by approximate computing, an hevc encoder case study. In Design, Automation \& Test in Europe Conference \& Exhibition (DATE), 494-499.

7. Oppenheim, A.V., \& Schafer, R.W. (1989). Discrete-Time Signal Processing. Englewood Cliffs, New Jersey: Prentice Hall.

8. Bhave, A.Y., \& Krogh, B.H. (2008). Performance bounds on state-feedback controllers with network delay. In 47th IEEE Conference on Decision and Control, 4608-4613.

9. Android's camera hardware abstraction layer, 2019.

10. Justusson, B.I. (1981). Median filtering: Statistical properties. In Two-Dimensional Digital Signal Processing II, Springer, Berlin, Heidelberg, 161-196.

11. Chen, C.Y., Choi, J., Gopalakrishnan, K., Srinivasan, V., \& Venkataramani, S. (2018). Exploiting approximate computing for deep learning acceleration. In Design, Automation \& Test in Europe Conference \& Exhibition (DATE), 821-826.

12. Matheron, G. (1974). Random Sets and Integral Geometry. New York: Wiley.

13. Baili, J., Marzougui, M., Sboui, A., Lahouar, S., Hergli, M., Bose, J.S.C., \& Besbes, K. (2017). Lane departure detection using image processing techniques. In IEEE 2nd International Conference on AntiCyber Crimes (ICACC), 238-241.

14. Kosecka, J., Blasi, R., Taylor, C.J., \& Malik, J. (1998). A comparative study of vision-based lateral control strategies for autonomous highway driving. IEEE International Conference on Robotics and Automation (Cat. No. 98CH36146), 3, 1903-1908.

15. Ragan-Kelley, J., Adams, A., Sharlet, D., Barnes, C., Paris, S., Levoy, M., \& Durand, F. (2017). Halide: Decoupling algorithms from schedules for high-performance image processing. Communications of the ACM, 61(1), 106-115.

16. Serra, J. (1982). Image Analysis and Mathematical Morphology. London: Academic.

17. Tukey, J.W. (1971). Exploratory Data Analysis. Reading, Massachusetts: Addison-Wesley.

18. Grabowski, K., \& Napieralski, A. (2010). Hardware architecture for advanced image processing. In IEEE Nuclear Science Symposuim \& Medical Imaging Conference, 3626-3633.

19. Anghel, L., Benabdenbi, M., Bosio, A., \& Vatajelu, E.I. (2017). Test and reliability in approximate computing. In International Mixed Signals Testing Workshop (IMSTW), 1-6.

20. Gallagher, N., \& Wise, G. (1981). A theoretical analysis of the properties of median filters. IEEE Transactions on Acoustics, Speech, and Signal Processing, 29(6), 1136-1141.

21. Maragos, P., \& Schafer, R.W. (1987). Morphological filters-Part I: Their settheoretic analysis and relations to linear shift-invariant filters. IEEE Transactions on Acoustics, Speech, and Signal Processing, 35(8), 1153-1169. 
22. Maragos, P., \& Schafer, R.W. (1987). Morphological filters-Part II: Their relations to median, orderstatistic, and stack filters. IEEE Transactions on Acoustics, Speech, and Signal Processing, 35(8), 11701184.

23. Borda, R.P., \& Frost Jr, J.D. (1968). Error reduction in small sample averaging through the use of the median rather than the mean. Electroencephalography and clinical neurophysiology, 25(4), 391-392.

24. Tyan, S.G. (1981). Median filtering: Deterministic properties. In Two-Dimensional Digital Signal Processing II, Springer, Berlin, Heidelberg, 197-217. 